\title{
A case of hypertrophic cardiomyopathy with "burned-out" apex of the left ventricle due to mid-ventricular obstruction
}

\author{
Elena Mershina $^{1}$, Olga Blagova ${ }^{2}$, Valentin Sinitsyn ${ }^{1}$ and Ekaterina Pershina ${ }^{1}$ \\ ${ }^{1}$ Federal Center of Treatment and Rehabilitation, Ivan'kovsloye sh.3, Moscow, 125367, Russia \\ ${ }^{2}$ I.M. Sechenov First Moscow State Medical University, 2-4 Bolshaya Pirogovskaya st., Moscow, 119991, Russia
}

\begin{abstract}
A rare case of mid-ventricular form of hypertrophic cardiomyopathy (HCM) with signs of LV apical aneurism and scar is presented. Coronary artery disease was excluded with help of coronary CTA. Cardiac MRI (CMR) with late gadolinium enhancement (LGE) revealed prominent apical fibrosis of LV with aneurysm formation due to mid-ventricular obstruction of LV cavity. Occurrence and pathophysiology of such HCM manifestations are discussed.
\end{abstract}

\section{Introduction}

Hypertrophic cardiomyopathy (HCM) is a relatively common cardiac disease of genetic origin with incidence about 1:500 in general population. It is remarkable that it is a cardiac imaging that plays a key role in diagnosis of this complex and heterogeneous heart disease of genetic origin. Cardiac MRI (CMR) is regarded as one of the most important imaging tools in such patients [1]. Besides 3-dimensional morphological and functional analysis of the heart with very accurate grading of location and extent of myocardial hypertrophy in patients with HCM, CMR with late gadolinium enhancement (LGE) revels presence and pattern of myocardial fibrosis in the myocardium. This biomarker has proven prognostic value in HCM patients. Nevertheless, some other atypical forms of myocardial hypertrophy and LGE patterns may be observed in HCM. The current case describes a rare variant of non-coronary LV apical post-ischemic lesions in patient with midventricular form of HCM.

\section{Case}

44 year-old woman complaining of some chest discomfort, rare episodes of palpitations and chest pain without relation to physical exercise was admitted to the hospital for diagnostic check-up. She has history of non-obstructive form of HCM diagnosed 2 years ago.

Physical examination found no abnormalities except for rare extrasystole. ECG demonstrated high-amplitude R V5-V6 with negative $\mathrm{T}$ waves, ST-elevation in V3-V4 leads which persisted for 2 last years. 24-hour Holter ECG recorded 1480 ventricular extrasystole, no runs of ventricular tachycardia.

Transthoracic echocardiography discovered prominent midventricular hypertrophy (Figure 1) without signs of left-ventricular (LV) outflow obstruction and presence of pressure gradient in the middle part of LV cavity. Apical akinesis was also found.

CMR was performed with 1.5 scanner (Magnetom Avanto, Siemens) using black blood techniques, cine- and phase-contrast MRI and LGE with double dose of $1.0 \mathrm{mmol} / \mathrm{ml}$ contrast agent (gadobutrol, Gadovist, Bayer AG). CMR confirmed presence of mid-ventricular LV hypertrophy and hypertrophy of papillary muscles (Figure 2). On systolic images LV cavity appeared obstructed in the middle part and it had «dumb-bell» appearance. Cine-MR detected presence of LV apical aneurysm with systolic bulging. CMR with LGE demonstrated

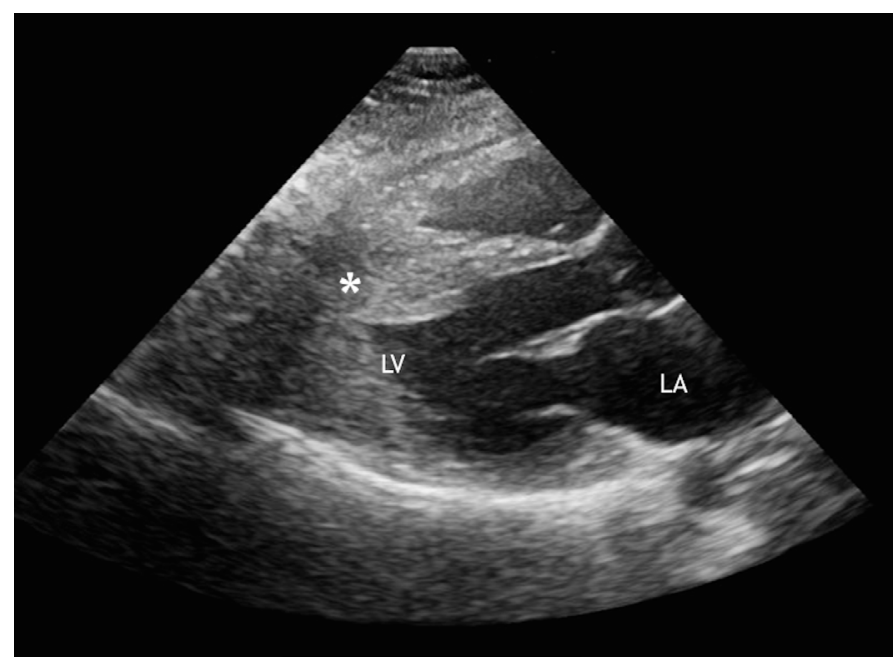

Figure 1. Echocardiography, parasternal view through LV outflow tract. Asterisk indicates middle part of hypertrophied IVS.

Correspondence to: Dr. Valentin Sinitsyn, Federal Center of Treatment and Rehabilitation, Ivan'kovsloye sh.3, Moscow, 125367, Russia, Tel: +7 495 9424020; E-mail: vsini@mail.ru

Key words: heart, myocardium, hypertrophy, hypertrophic cardiomyopathy, MRI, fibrosis, ischemia, scar

Special Issue: Imaging of myocardial diseases: to new horizons

Valentin E. Sinitsyn

Professor

Department of Radiology

Federal Center of Medicine and Rehabilitation

Russia

E-mail: vsini@mail.ru

Published: July 30, 2016 
an ischemic pattern of contrast enhancement (subendocardial and transmural) of the myocardium in the apical region (Figure 3). Such type of contrast enhancement is regarded as not typical for HCM. In order to exclude the presence of coronary artery disease, a lowdose coronary CT-angiography (CTA) was done (Discovery 750, GE Healthcare). CTA confirmed absence of obstructive coronary disease: no coronary calcifications or soft plaques in the coronaries. There was a myocardial bridge over proximal segment of left anterior descending artery (LAD) (Figure 4).

\section{Discussion}

It is well known that the wide genetic heterogeneity of HCM manifest itself with wide variations in morphologic expression of the disease. Asymmetric myocardial hypertrophy of the interventricular septum (IVS) is the most common variant of HCM [2]. It can be accompanied with obstruction of LV outflow tract (accordingly, there are obstructive and non-obstructive variants of HCM). More rare variants of HCM are ones with symmetric, apical or mass-like hypertrophy. Right ventricular involvement may occur. Increasing popularity of CMR in
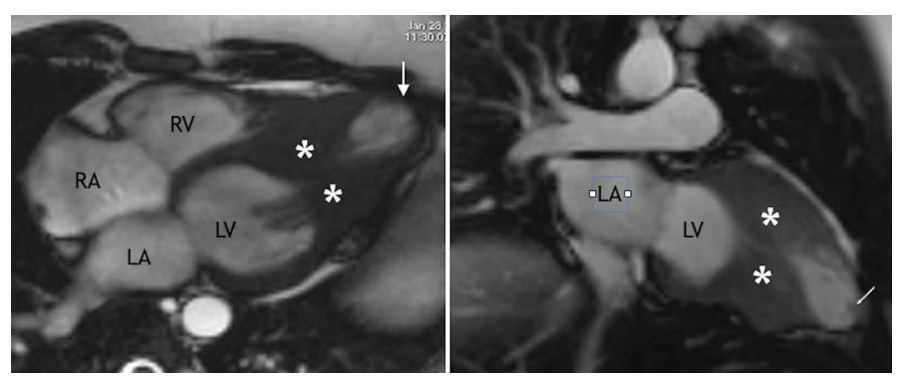

Figure 2. CMR. Left side - long-axis view, right side - vertical long-axis two-chamber view. Site of mid-ventricular obstruction is indicated with asterisks, apical aneurysm is shown with arrow.

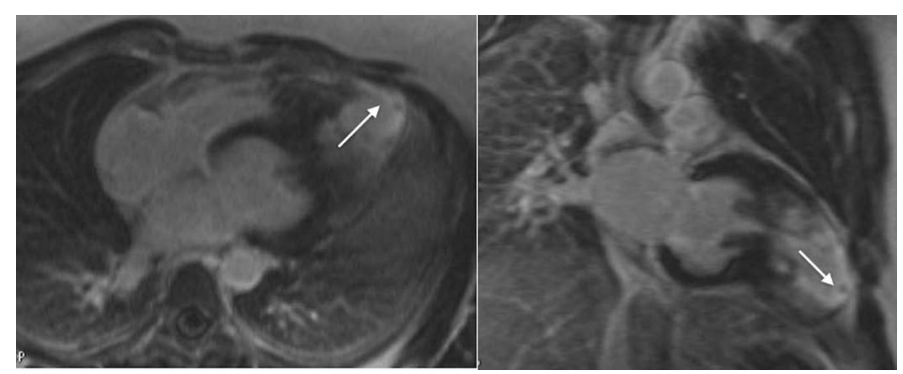

Figure 3. LGE CMR. Left side - long-axis view, right side vertical long-axis two-chamber view. Area of subendocardial and transmural contrast enhancement in the apical segments of LV myocardium is marked with arrow.

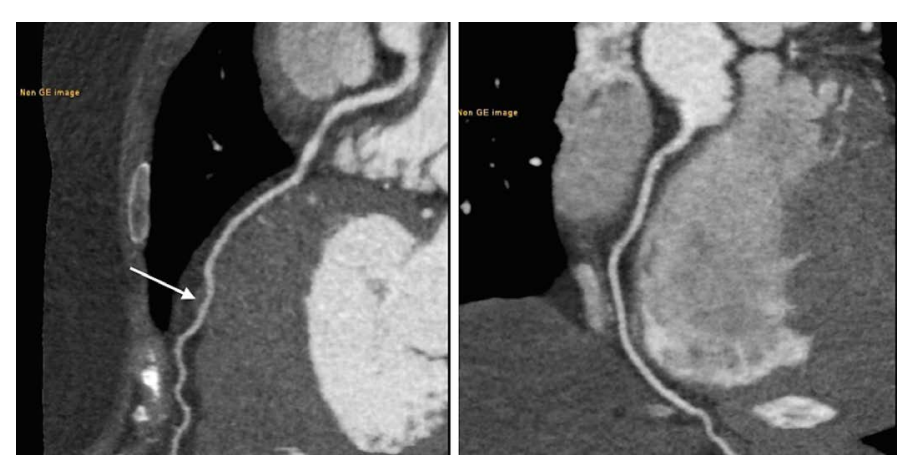

Figure 4. CTA, vessel views. Left side - normal LAD with myocardial bridge over it (an arrow). Right side - normal RCA. imaging of patients with HCM is based on several advantages of this technique [1-3]. Accurate 3D quantification of LV hypertrophy and unique capability for myocardial characterization with help of LGE or T1-mapping are probably the most important ones. Presence of LGE in HCM usually indicates to myocardial fibrosis. HCM patients with LGE more prone to malignant forms of ventricular arrhythmias and generally they have worse prognosis. Several patterns of LGE in HCM have been described, but they are different from so-called ischemic (gradient of LGE from endocardium to epicardium) pattern. This is CMR with LGE has an important role in differentiation of HCM from other diseases accompanied with LV myocardial hypertrophy. Most often LGE MRI shows intramyocardial foci of contrast enhancement inside the hypertrophied segments.

This case is interesting for several reasons. First of all, echocardiography and CMR revealed mid-ventricular level of blood flow obstruction inside the LV cavity (usually LV obstruction in HCM is located (if present) in the outflow tract of LV). Secondly, LGE MRI discovered an unusual patter of myocardial enhancement. It needs more detailed explanation. In the presented case the patient had prominent mid-ventricular hypertrophy together with hypertrophy of papillary muscles. These factors caused LV mid-cavity obstruction with a 'dumb-bell' configuration of ventricular cavity. In severe cases of mid-ventricular narrowing and obstruction an apical dilatation of LV arises. The apical dilatation may develop due to increased systolic pressure inside the «distal» part of LV cavity during dynamic periods of systolic obstruction. Such long-term pressure overload and strain of apical myocardium together with small vessel intramural coronary artery disease which is present in some patients with HCM noncoronary myocardial necrosis and prominent fibrosis may occur [4-6]. Accordingly, LGE MRI shows contrast enhancement of apical myocardium (a syndrome of "burned-out apex") which sometimes should be differentiated from antero-septal chronic myocardial infarction with formation of apical aneurysm. In the presented case combined use of echocardiography, CMR and CTA allowed for differentiation of mid-ventricular form of HCM with syndrome of "burned-out" apex from apical myocardial infarction, apical form of HCM or Tako-Tsubo syndrome.

\section{Conclusion}

In HCM patients having mid-ventricular obstruction prominent fibrotic changes of the apical myocardium may occur. On LGE CMR they look like apical LV aneurysm. Such pattern of LV myocardial contrast enhancement is caused by ischemia resulting from increased myocardial strain, reduced myocardial capillary density, small-vessel intramural coronary artery disease and increased perivascular fibrosis but not by stenotic lesions in the large epicardial coronary arteries. CMR assisted with CTA helps to diagnose this rare pathology.

\section{References}

1. Maron BJ, Maron MS (2016) The Remarkable 50 Years of Imaging in HCM and How it Has Changed Diagnosis and Management: From M-Mode Echocardiography to CMR. JACC Cardiovasc Imaging 9: 858-872.[Crossref]

2. Hansen MW, Merchant N (2007) MRI of hypertrophic cardiomyopathy: part 2 , Differential diagnosis, risk stratification, and posttreatment MRI appearances. AJR Am J Roentgenol 189: 1344-1352.[Crossref]

3. Noureldin RA, Liu S, Nacif MS, Judge DP, Halushka MK, et al. (2012) The diagnosis of hypertrophic cardiomyopathy by cardiovascular magnetic resonance. J Cardiovasc Magn Reson 14: 17.[Crossref]

4. Moon JC, Fisher NG, McKenna WJ, Pennell DJ (2004) Detection of apical hypertrophic cardiomyopathy by cardiovascular magnetic resonance in patients with non-diagnostic echocardiography. Heart 90: 645-690.[Crossref] 
Mershina E (2016) A case of hypertrophic cardiomyopathy with "burned-out" apex of the left ventricle due to mid-ventricular obstruction

5. Nakamura T, Matsubarra K, Furukawa K (1992) Diastolic paradox jet flow in patients with hypertrophic cardiomyopathy: evidence of concealed apical asynergy with cavity obliteration. J Am Coll Cardiol 19: 516-524. [Crossref]
6. Po JR, Kim B, Aslam F, Azuma A, Sugihara H, et al. (2015) Doppler systolic signal void in hypertrophic cardiomyopathy: apical aneurysm and severe obstruction without elevated intraventricular velocities. J Am Soc Echocardiogr 28: 1462-1473. [Crossref]

Copyright: (C2016 Mershina E. This is an open-access article distributed under the terms of the Creative Commons Attribution License, which permits unrestricted use, distribution, and reproduction in any medium, provided the original author and source are credited. 